\title{
Perceived Research Environment and Research Burnout in Indonesian Academics: The Moderator Role of Research Importance
}

\author{
Dian R. Sawitri* \\ Faculty of Psychology, Diponegoro University \\ Jl. Prof. Soedarto, S.H., Tembalang,Semarang 50275, Indonesia \\ *Dian. R. Sawitri, mobile: +628/ /2798878, email: dian.r.sawitri@gmail.com
}

\begin{abstract}
Research burnout is an individual's emotional condition correlated with long-term pressures in the processes of academic research and publication. Nowadays, involve in more research activities in order to be more research productive is expected among higher education academics. These expectations put a lot of pressure on higher education academics to publish and also to generate grant money. Those who cannot meet the expectations may feel a sense of research burnout. We surveyed 290 Indonesian academics ( $49.8 \%$ female, mean age $=42.30$ years) from several higher degree institutions in Indonesia. We used scales of research importance, perceived research environment, and research burnout. Hierarchical regression analyses demonstrated that research importance moderated the relationship between perceived research environment and research burnout. For those who have low research importance, perceived research environment was positively correlated with burnout, but for those who have high research importance, perceived research environment was negatively correlated with burnout.
\end{abstract}

Keywords: Research burnout, academics, research importance, perceived research environment

Received 2 January 2020/Accepted 27 May 2020 CJEHCP All rights reserved

\section{Introduction}

The three main functions which chraracterized the academic profession in higher education systems, including education, research, and services (Eam, 2005). However, academic research and scientific publications have been more and morehighlighted at most universities (Brew \& Lucas, 2009). Previous studies have shown that engagement in research activities potentially advances teaching quality (Brew \& Boud, 1995) and improves knowledge and competence that contributes to high quality supervision in research (Lindsay, Breen, \& Jenkins, 2002). Accordingly, there has been a consistent tendency for universities in developed countries to improve their focus on research. This trend has reached to developing countries, where research activities and outcomes are 
increasingly considered as a main concern (Nguyen, Klopper, \& Smith, 2016). As academics are required to be research productive, research-related demands can potentially be a burden and lead to burnout (Singh, Mishra, \& Kim, 1998). The major feature of burnout is that individual cannot or will not do again what one has been doing (Levinson, 198I).

There is an evidence that research burnout lead to lower job satisfaction (Singh et al., 1998). Previous studies have also demonstrated various antecedents of burnout in academics or faculty members, for example, number of students taught, time allocated in a variety of activities, and student evaluation (Lackritz, 2004), job control, demands, and work self-determination (Fernet, Guay, \& Senecal, 2004). However, predictors of research-related burnout have not been widely explored. Singh et al. (1998) demonstrated that lower intrinsic motivation for conducting research and lack of contingent reward predicted research-related burnout.

Among the antecedents of research-related outcomes, environmental factors take a big part in explaining it (Bland \& Ruffin, 1992), and this fact leads researchers to recognize the characteristics a good environment for doing research (Wilkes \& Jackson, 2003). Previous studies showed that a good research culture and environment leads to higher engagement in research (e.g., Howard, Ferguson, Wilkinson, \& Campbell, 2013; Whelan, Copeland, Oladitan, Murrells, \& Gandy, 20I3).

More recently, Sawitri, Creed, Nurtjahjanti, and Prasetyo (2020) created a new scale to measure percieved research environment for academics. Perceived research environment was defined as the organisational environment perceived by academics, which allows and facilitates them to learn and conduct research activities and producescientific publications. It contains five domains ofbeneficial social relationships (i.e., the condition where individuals perceive that their social relationships as supportive to their involvement in research-related activities), positive reinforcement (i.e., the confdition where individuals perceive positive social and non-social reinforcement for attainments in research-related activities), support and expectations (i.e., perceived university support and expectations for doing research-related activities), institutional emphasis on research (i.e., perceived highlights on research-related activities), and positive role models (i.e., perceived availability of encouraging role model for doing research activities). Perceived research environment was found 
to be associated positively with organisational culture/support for conducting research and research involvement in academics (Sawitri et al., 2020).

The fit between individual and one's environment is in line with the interactionist theory of behaviour, which underlines that the interaction between the individual and the environment is responsible for the variance in behavioural and attitudinal variables (Muchinsky \& Monahan, 1987). The contemporary theories of person-environment fit involves three basic assumptions: (a) individuals tend to find environments that correspond with their characteristics; (b) the degree of individual-environment fit is correlated with important outcomes for not only the individual but also the environment. Greater fit tends to produce positive outcomes, such as satisfaction, enhanced performance, and stronger persistence, whereas poorer fit correlates with negative outcomes, such as dissatisfaction and low levels of achievement; and (c) the process of fit is bi-directional, as the individual can give an effect to the environment, and the environment may influence the individual. This relationship represents a system, where change in one aspect influences the other aspect (Swanson \& Fouad, 1999).

Applied to the organisational context, person-organisation fit is the compatibility between individuals and organisations that occurs when at least one entity supports what the other needs (complementary fit), or they share similar basic characteristics (supplementary fit), or both (Kristof, 1996). First, complementary fit involves needs-supplies fit, referring to the condition that happens when the needs of the individual are fulfilled by the support from others in the organisation; and demands-abilities fit, referring to the condition that is achieved when the individual has the ability to satisfy the demands of the organisation. Second, supplementary fit involves the similarity of characteristics between the individual and the organisation.

Reflecting this, individual's characteristic needs to be considered in predicting research burnout, such as goal importance (cf. Latham \& Locke, 199I).When the goal is perceived as an important aspiration, it will stimulate negative emotions (Latham \& Locke), and in turn, arouse more strategy regulatory responses. In contrast, individuals will not use regulatory effort if the goal is not on their top priority (Hollenbeck \& Williams, 1987).Individuals are active agents who created their own lives by constantly formulating and accomplishing goals that they consider can lead them to their final 
destination (Bandura, 1991). Several studies demonstrated the role of goal importance in moderating perceived environment influence and outcomes (e.g., Hu, Creed, \& Hood, 20I8).

This research is unique for several reasons. First, we examined the correlates of perceived research environment. In measuring perceived research environment, we used a newly developed scale, which was designed based on the experience of Indonesian academics (Sawitri et al., 2020).Second, previous studies have explored burnout in university faculty members (e.g., Alves, Oliveira, \& Paro, 2019; Singh, Jain, \& Kaur, 2017; Sabagh, Hall, \& Saroyan, 2018). However, the correlates of research-related burnout are rarely investigated. This research filled this gap by examining the antecedent variables of research-related burnout.

We operationalize goal importance as research importance as having a research importance represents having research-related goals and activities that are important and meaningful for the individual. Our hypotheses were that for those who have high research importance, perceived research environment would be associated negatively with research burnout, whereas for those with low research importance, perceived research environment would be associated positively with research burnout.

\section{Methods}

Participants

Participants were 290 academics (49.8\% female, 3.8\% did not report gender, mean age $=42.30$ years, $S D$ age $=9.86 ; 56.2 \%$ did not report age) from several higher degree institutions in Indonesia who were recruited using convenience sampling. Prior to completing the survey, participants fill in a consent form agreeing to their participation in the study. A large majority (65.9\%) reported that they held a masters' degree, and $21.6 \%$ held a doctorate ( $9.2 \%$ did not report education level). A small percentage of them (I.7\%) were professors, $23.4 \%$ associate professors, $30 \%$ assistant professors, $13.8 \%$ lecturers, $4.5 \%$ were junior lecturers $(27.8 \%$ did not provide information regarding their position). Mean tenure was 16.54 years $(S D=9.54 ; 14.8 \%$ did not reveal information on tenure). 
Materials

The survey booklet consisted of measures of perceived research environment, research important, research burnout. All of the scales used a 6-point Likert-like scale $(I=$ strongly disagree and $6=$ strongly agree). Higher totals cores indicate higher levels of each construct.

Perceived Research Environment. The 25-item Perceived Research Environment Scale (Sawitri et al., 2020) was used to assess perceived research environment in academicswhich gives opportunity and assists them to learn and conduct research activities and produce scientific publications, and includes five domains of beneficial social relationship, positive reinforcement, support and expectations, focus on research, and positive role models.Sample item is "Many academics at my university are working on important research projects," on a 6-point scale. Alpha has been reported as .92, and Construct validity has demonstrated by expected correlations with measures of organisational culture/support and research involvement (Sawitri et al., 2020).

Research Importance. The 6-item Research Importance Scale, containing the 3-item Goal Importance Scale (Sideridis, 200I) and three items from the 9-item Goal Commitment Scale (Hollenbeck, Williams, \& Klein, 1989) was used to measure the level of perceived research importance. Sample items: "The highest priority for me right now is to study hard to achieve high GPA" was modified into "The highest work priority for me right now is to conduct research in my area of interests," and "I am strongly committed to pursuing this goal" was changed into "I am strongly committed to conducting research in my area of interest."

Research Burnout. The 6-item Research-related Burnout Scale (Singh et al., 1998) was used to measure an emotional condition associated with long-term pressures in the academic research and scientific publication processes. Sample item: "I have frequently thought about quitting academic research. "Alpha has been reported as .82 - .85 and construct validity has been supported by negative correlation with intrinsic motivation for conducting research and job satisfaction scales, and positive correlation with minimum performance-contingent rewards (Singh et al.). 


\section{Translation}

We followed Jones, Lee, Phillips, Zhang, and Jaceldo's (200I) recommendations to translate the measures into Bahasa Indonesia: (a) the items were translated into Bahasa Indonesia by the first author, who is bilingual; (b) the translated version wassubsequently examined by two monolingual Indonesian academics; (c) two native bilingual Indonesian academicsblindly back-translated the items into English; (d) the back-translated items were subsequently compared with the original English version by all authors to makes sure that the meaning was accurate, and any mistakes were adjusted; and (e)three Indonesian academics examined the final Indonesian version to ensure the readability.

\section{Procedure}

Ethical approval was granted by the ethics committeeref. no. 2017/1006. The survey was administered by the research assistants during working hours.

\section{Data Analyses}

Hierarchical regression analyses were used to examine whether research importance moderated the relationship between perceived research environment and research burnout. Perceived research environment was included at Step I, research importance at Step 2, and the interaction term (i.e., perceived research environment $x$ research importance; Jose, 2013) at Step 3.

\section{Results}

First of all, we conducted a confirmatory factor analysis (CFA; AMOS) to check whether all items loaded onto their respective latent factor and that all latent factors were independent. The first model involved the scales of perceived research environment, research importance, and research burnout. This model demonstrated satisfactory fit statistics, $\chi^{2}(\mathrm{df}, \mathrm{N}=290), \chi^{2} / \mathrm{df}<3.0$, CFI and TLI values $=.93$, and RMSEA $=.07$ (Hair, Black, Babin, \& Anderson, 2010), and all factors loading were significant $(p<.0 \mathrm{I}$; range $-.19-.47)$. Bivariate and latent variable correlations are displayed in Table I. 
Table I

Intercorrelations, means, standard deviations, and Cronbach Alphas; $N=290$

\begin{tabular}{lcccccc}
\hline \multicolumn{1}{c}{ Variables } & $\mathrm{I}$ & 2 & 3 & $\mathrm{M}$ & $\mathrm{SD}$ & $\alpha$ \\
\hline Perceived research environment & - & $.44^{* *}$ & -.08 & $\mathrm{III} .63$ & 15.53 & .92 \\
Research importance & $.47^{* *}$ & - & $-.18^{* *}$ & 28.75 & 5.15 & .70 \\
Researh burnout & $.10^{* *}$ & $-.19^{* *}$ & - & 16.67 & 7.23 & .94
\end{tabular}

Note. Correlations among scales above diagonal; correlations among variables below

Subsequently, hierarchical regression analyses were applied to examine the moderated effects. The perceived research environment scale was entered at Step I, and accounted for $0.7 \%$ of the variance in research burnout, but the model was not significant, $F(I, 288)=2.003, p=.16$. Perceived research environment was not associated with research burnout $(\beta=-.08, p=.16)$. Research importance at Step 2 accounted for a further $2.4 \%, F(I, 287)=7.20, p<.05$. Higher research importance $(\beta=-.17, p=.01)$ was associated significantly with lower research burnout. At Step 3, the interaction term was significant, $F(I, 286)=10.01, p=.01$, and $6.4 \%$ of the variance in research burnout was accounted for. See Table 2 .

Table 2

Hierarchical regression analysis $(N=290)$

\begin{tabular}{lcccc}
\hline \multicolumn{1}{c}{ Variables } & \multicolumn{4}{c}{ Research Burnout } \\
\hline \multicolumn{1}{l}{$\begin{array}{l}\text { Step I } \\
\text { Perceived research environment } \\
\text { (PRE) }\end{array}$} & .007 & -.04 & -.08 & -.08 \\
$\begin{array}{l}\text { Step 2 } \\
\text { Research importance (RI) }\end{array}$ & & & & \\
Step 3 & $.02^{*}$ & -.24 & -.17 & $-.17^{* *}$ \\
\hline PRE x RI interaction & & & & \\
\hline
\end{tabular}

The results demonstrated that for those who have high research importance, perceived research environment would be associated negatively with research burnout, whereas for those with low research importance, perceived research environment would be associated positively with research burnout, as perceived research environment was seen as a pressure for doing research. 


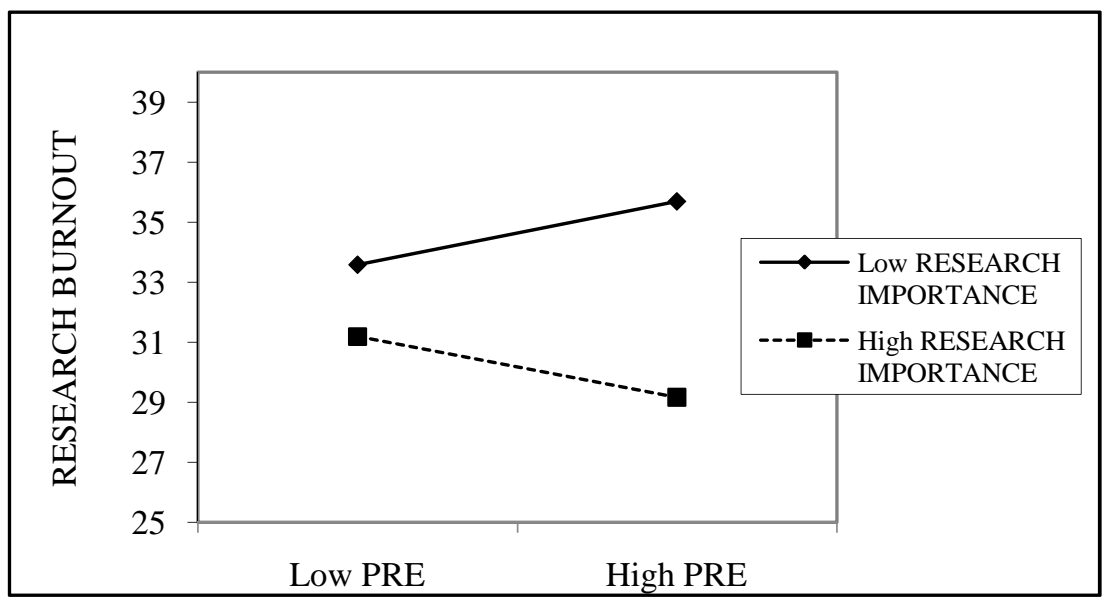

Figure I. Research importance moderated the relationship between perceived research environment (PRE) and research burnout

\section{Discussion}

In this study, we tested a model based on person-organisation fit theory. We proposed that research importance served as a moderator variable in the relationship between perceived research environment and research burnout. We found non-significant relationship between perceived research environment and research burnout, and found that the relationship between perceived research environment and burnout was negative when research importance was higher. This suggest that, those who have high research importance are more likely to see perceived research environment as facilitative in reducing research burnout. This is consistent with Swanson and Fouad's (1999) perspective that greater person-environment fit leads to positive outcomes, such as higher satisfaction, better performance, and lower stress.

We also found that when research importance was lower, greater perceived research performance led to higher research burnout. This indicates that those who have low research importance tend to see perceived research environment as an additional load leads to research burnout. This is also in line with Muchinsky and Monahan's (1987) perspective that interaction between the individual and the environment has a great impact on behavioural and attitudinal variables. 
The findings of this study were consistent with previous research by Lackritz (2014) in 265 university faculty members in the US, which demonstrated several predictors of burnout, including publication-relation, teaching, and other activities. Similar results were also found in Sabagh et al.'s (2018) study which explored antecedent variables of burnout. These researchers found researchrelated stressors may contribute to burnout in faculty members.

Our findings have several implications. First, interventions aimed at assisting academics to reduce research burnout might include a focus on increasing research importance, as the role of perceived research environment cannot stand alone. This kind of intervention would allow academics with low research importance to commit more on research-related activities and relieve researchrelated burden. Second, research environment could be created to be more academics friendly, so that it will help them focusing more on research voluntarily.

Conclusively, our study extended current knowledge by underlining the role play by research importance on research burnout in academics and made a contribution to the academics' career development by showing that the relationship between perceived research environment and research burnout are conditional upon the degree of research importance.

Our study involved a sample of higher education academics from several universities in Central Java, Indonesia. Therefore, the conclusions of this study need to be examined on other academic populations. The data were collected at one point in time, therefore, future studies could focus on testing the longitudinal associations among the study variables to obtain more robust causal inferences. Finally, although we found significant interaction effect, the variance explained was very modest. This suggests that other individual variables might play a rolein moderating the relationship, and other contextual variables need to be examined.

\section{References}

Alves P.C., Oliveira, A. d. F, Paro, H. B. M. S. (2019). Quality of life and burnout among faculty members: How much does the field of knowledge matter? PLoS ONE, 14,I - 12. doi:10.137|/journal.pone.0214217. 
Bandura, A. (199I). Social cognitive theory of self-regulation. Organizational Behaviour and Human Decision Processes, 50, 248-287. doi:10.1016/0749-5978(9I)9022-L.

Bland, C. J., \& Ruffin, M. T. (1992). Characteristics of a productive research environment: Literature review. Academic Medicine, 67, 385-397. doi:10.1097/0000 I888-199206000-000-10.

Brew, A., \& Boud, D. (1995). Teaching and research: Establishing the vital link with learning. Higher Education, 26, 26I-273. doi:I0.1007/BF0I 384493.

Brew, A., \& Lucas, L. (2009). Introduction: Academic research and researchers. In A. Brew, \& L. Lucas (Eds.). Academic research and researchers. New York, NY: Open University Press.

Carver, C. S., \& Scheier, M. F. (1990). Origins and functions of positive and negative affect: A control process view. Psychological Review, 97, 19-35. doi:10.1037/0033-295X.97.1.19.

Eam, P. (2015). Investigating relationship among research self-efficacy, research outcome expectations, and research interest of Cambodian faculty: Testing social-cognitive theory. International Journal of Sociology of Education, 4, 199-224. doi:I0.I7583/rise.20I5. 1752.

Fernet, C., Guay, F., \& Senecal, C. (2004). Adjusting to job demands: The role of work selfdetermination and job control in predicting burnout. Journal of Vocational Behavior, 65, 39-56. doi:I0.1016/S000I-879I(03)00098-8.

Hollenbeck, J. R., \& Williams, C. L. (1987). Goal importance, self-focus, and the goal-setting process. Journal of Applied Psychology, 72, 204-2II. doi:I0.1037/002I-9010.72.2.204.

Hollenbeck, J. R., Williams, C. L., \& Klein, H. J. (1989). An empirical examination of the antecedents of commitment to difficult goals. Journal of Applied Psychology, 74, 18-23.

Howard, A. J., Ferguson, M., Wilkinson, P., \& Campbell, K. L. (20I3). Involvement in research activities and factors influencing research capacity among dietitians. Journal of Human Nutrition and Dietietics, 26, I80-187. doi:10.1 III/jhn. 12053

Hu, S., Creed, P. A., \& Hood, M. H. (2018). Career goal importance as a moderator in the relationship between career feedback and career-related stress. Journal of Career Development, 45, 3-I8. doi:I0.1 I77/08948453/6667847.

Jones, P. S., Lee, J. W., Phillips, L. R., Zhang, X. E., \& Jaceldo, K. B. (200I). An adaptation of Brislin's translation model for cross-cultural research. Nursing Research, 50, 300-304. doi:10.1097/00006199-200109000-00008.

Jose, P. E. (20/3). Doing statistical mediation and moderation. New York, NY: The Guildford Press.

Kristof, A. L. (1996). Person-organization fit: an integrative review of its conceptualizations, measurement, and implications. Personnel Psychology, 49, I-49.doi:10.1 III/j.17446570.1996.tb0I790.x 
Lackritz, J. R. (2004). Exploring burnout among university faculty: Incidence, performance, and demographic issues. Teaching and Teacher Education, 20, 713-729. doi:I0.10I6/j.tate.2004.07.0002.

Latham, G. P., \& Locke, G. A. (199I). Self-regulation through goal-setting. Organizational Behavior \& Human Decision Processes, 50, 212-247. doi:10.1016/0749-5978(91)90021-K.

Levinson, H. (198I). When executive burnout. Harvard Business Review, 59, 73-8I.

Lindsay, R., Breen, R., \& Jenkins, A. (2002). Academic research and teaching quality. The views of undergraduate and postgraduate students. Studies in Higher Education, 27, 309-327. doi: 10.1080/03075070220000699.

Muchinsky, P. M., \& Monahan, C. J. (1987). What is person-environment congruence? Supplementary versus complementary models of fit. Journal of Vocational Behavior, 3I, 268-277. doi: $10.1016 / 0001-879 \mid(87) 90$.

Nguyen, Q., Klopper, C., \& Smith, C. (2016). Affordances, barriers, and motivations: Engagement in research activity by academics at the research-oriented university in Vietnam. Open Review of Educational Research, 3, 68-84. doi:10.1080/23265507.2016.II 70627.

Sabagh, Z., Hall, N. C., \& Saroyan, A. (2018) Antecedents,correlates and consequences of faculty burnout. Educational Research, 60, I3I-I56, doi:10.1080/0013188I.2018.1461573.

Sawitri, D. R., Creed, P. A., Nurtjahjanti, H., \& Prasetyo, A. R. (2020). Development and initial validation of perceived research environment scale for higher education academics. Journal of Psychoeducational Assessment, 38, 195 - 208. doi:10.1 I77/0734282919828892.

Sideridis, G. D. (200I). The causal role of goal importance for the explanation of student's study behaviour: Cross-validation with multiple samples. Educational Psychology, 21, 277-298. doi:10.1080/0|4434I0I20065487.

Singh, I., Jain, M., \& Kaur, R. (2017). A study of level of stress and burnout among faculty members in private universities of Punjab. International Journal of Applied Business and Economic Research, 15, $435-446$.

Singh, S. N., Mishra, S., \& Kim, D. (1998). Research-related burnout among faculty in higher education. Psychological Report, 83, 463-473.

Swanson, J. L., \& Fouad, N. A. (1999). Applying theories of person-environment fit to the transition from school to work. Career Development Quarterly, 47, 337-347. doi:10.1002/j.21610045.1999.tb00742.x

Whelan, K., Copeland, E., Oladitan, L., Murrells, T., \& Gandy, J. (20I3). Development and validation of a questionnaire to measure research involvement among registered dietitians. Journal of the Academy of Nutrition and Dietetics, I I3, 563-568. doi: I0.10 I6/j.jand.2012.08.027. 
Wilkes, L., \& Jackson, D. (20/3). Enabling research cultures in nursing: Insights from a multidisciplinary group of experienced researchers. Nursing Research, 20, 28-34. 